\title{
Application of computational fluid dynamics in piping distribution system and special focus on the Arabian peninsula: a review
}

\begin{abstract}
Drinking Water Distribution Networks are the life line for urban population. Disruption in services can cripple the system. In order to supply potable water, the authorities must stick to WHO guidelines. The major problems associated are contaminants, leakage and water hammer effects. The CFD studies discussed in the current paper have shown to provide, an insight on dealing with these issues. Further studies need to be carried out in order to properly use the available tools. An integration of the available CFD tools with EPANET would be the right way to move forward. The majority of the researchers have implemented CFD in order to study the behavior of mixing, leakage and water hammer in WDN, related to North American and European climatic conditions. The current review aims to compile the application of Computation Fluid Dynamics in the water distribution networks.
\end{abstract}

Keyword: CFD; Drinking water; Pipeline distribution; Corrosion; Leaks 